\title{
DESEMPENHO DE TESTES DE NORMALIDADE MULTIVARIADOS AVALIADO POR SIMULAÇÃO MONTE CARLO
}

\author{
Monte Carlo evaluation of the performance of multivariate normality tests
}

\author{
Narjara Fonseca Cantelmo ${ }^{1}$, Daniel Furtado Ferreira ${ }^{2}$
}

\begin{abstract}
RESUMO
Neste trabalho, objetivou-se avaliar a performance do teste multivariado de normalidade de Shapiro-Wilk implementado no $\mathbf{R}$ comparando o seu desempenho com os testes de assimetria e curtose de Mardia (1970,1974, 1975) utilizando simulação Monte Carlo. Foram mensuradas e comparadas as taxas de erro tipo I e poderes dos testes. Pode-se concluir que o teste de Shapiro-Wilk multivariado do programa $\mathbf{R}$, função mshapiro.test do pacote mvnormtest, tem fraco desempenho (liberal) e não é recomendado para uso rotineiro.
\end{abstract}

Termos pra indexação: Shapiro-Wilk, assimetria, curtose, poder, erro tipo I.

\begin{abstract}
This work aimed to evaluate the performance of the multivariate normality test of Shapiro-Wilk implemented in $\mathbf{R}$ in the library mvnormtest and to compare it with the asymmetry and kurtosis normality test proposed by Mardia $(1970,1974,1975)$ using Monte Carlo simulation. The multivariate normality test of Shapiro-Wilk is not recommended for regular use.
\end{abstract}

Index terms: Shapiro-Wilk, asymmetry, kurtosis, power, type I error rate.

(Recebido em 19 de março de 2007 e aprovado em 10 de julho de 2007)

\section{INTRODUÇÃO}

A suposição de normalidade dos dados amostrais ou experimentais é uma condição exigida para a realização de muitas inferências válidas a respeito de parâmetros populacionais. Vários dos diferentes métodos de estimação e testes de hipóteses existentes foram formulados sob a suposição de que a amostra aleatória tenha sido extraída de uma população normal. Da mesma forma que ocorre no caso univariado, têm-se inferências multivariadas sobre parâmetros que são vetores ou matrizes, pois a estatística multivariada é lida com observações simultâneas de várias variáveis. Os testes de hipóteses e os métodos de estimação são, em geral, baseados na suposição de normalidade multivariada da amostra aleatória.

A necessidade de testar a hipótese de normalidade multivariada fica evidenciada quando o pesquisador pretende avaliar se as condições pressupostas para a validade da inferência que irá realizar foram atendidas. A existência de um teste adequado, com propriedades ótimas sempre foi questionada. Um destes testes de normalidade é baseado nos desvios de assimetria e curtose (MARDIA, 1970, 1974, 1975). Alguns problemas podem ser destacados desta abordagem. A princípio, a não rejeição da hipótese de distribuição simétrica e mesocúrtica não garante que a distribuição seja realmente normal. Existem contra-exemplos de distribuições não-normais simétricas e mesocúrticas. A segunda dificuldade é a propriedade assintótica das estatísticas dos testes. As distribuições destas estatísticas são apenas assintoticamente válidas, o que dificulta a aplicação dos testes em conjuntos de dados de tamanhos relativamente pequenos.

Um teste multivariado para normalidade foi proposto por Royston (1983) generalizando o teste univariado de Shapiro-Wilk para o caso multivariado. A justificativa apresentada para a proposição deste teste refere-se ao fato de que muitos testes multivariados possuem distribuições matematicamente intratáveis sob a hipótese nula para a estatística. No entanto, as propriedades do teste de Shapiro-Wilk generalizado para o caso multivariado não foram avaliadas por simulação, principalmente para dimensões dos vetores das variáveis aleatórias maiores do que três.

O programa $\mathbf{R}$ (R DEVELOPMENT CORE TEAM, 2006) tem tido grande impacto no meio científico e por ter código fonte aberto, tem recebido contribuições de pesquisadores de todo o mundo. O pacote mvnormtest e a

\footnotetext{
${ }^{1}$ Aluna do curso de Agronomia - Departamento de Ciências Exatas/DEX - Universidade Federal de Lavras/UFLA - Cx. P. 3037 - $37200-000$ - Lavras, MG nacantelmo@hotmail.com - Bolsista CNPq

${ }^{2}$ Pós-Doutorado, Professor - Departamento de Ciências Exatas/DEX - Universidade Federal de Lavras/UFLA - Cx. P. 3037 - $37200-000$ - Lavras, MG danielff@ufla.br - Bolsista CNPq
} 
função mshapiro.test deste pacote possibilitam ao usuário aplicar o teste de normalidade multivariada de ShapiroWilk que não trata da extensão multivariada de Royston (1983). Esta função é baseada na generalização multivariada do teste proposto por Domanski (1998) que se baseia em buscar uma combinação linear das $p$ variáveis originais e aplicar o teste de Shapiro-Wilk nesta nova variável. O procedimento de Royston (1983) prevê a estimação da estatística W de Shapiro-Wilk para cada uma das variáveis, sendo a estatística final do teste baseada na soma dos seus valores. É utilizada uma transformação da estatística e a correlação entre as variáveis é utilizada para obter os graus de liberdade da distribuição de qui-quadrado resultante (ROYSTON, 1983).

Uma forma de avaliar o desempenho de um teste é mensurar tanto as taxas de erro tipo I, em diferentes condições da hipótese nula de normalidade, quanto o poder do teste, simulando amostras sob a hipótese alternativa de não-normalidade. Um teste ideal, apesar de não existir, seria aquele que não rejeitasse para nenhuma amostra observada a hipótese nula verdadeira e rejeitasse $100 \%$ das vezes as hipóteses nulas falsas. Como isso não ocorre em situações reais, busca-se um teste que mantenha as taxas de erro tipo I menores ou iguais a um valor nominal de probabilidade escolhido, diga-se, $\alpha$, e que tenha o maior poder possível. O poder é a probabilidade de rejeitar a hipótese nula quando ela é realmente falsa.

A motivação para este trabalho surgiu a partir do interesse de avaliar o desempenho do teste multivariado de normalidade de Shapiro-Wilk implementado no R. Assim, foi proposto este trabalho objetivando-se comparar o desempenho do teste multivariado de normalidade de Shapiro-Wilk com o teste de assimetria e curtose de Mardia utilizando simulação Monte Carlo.

\section{MATERIAL E MÉTODOS}

Duas estratégias foram consideradas neste trabalho. A primeira teve o intuito de avaliar as taxas de erro tipo I dos testes de Shapiro-Wilk e de assimetria e curtose de Mardia. A segunda foi delineada para avaliar o poder destes testes. Em ambos os casos foi utilizada simulação Monte Carlo. Em cada simulação foram aplicados os testes de normalidade em um nível nominal préestabelecido de significância, sendo verificado se a hipótese nula foi ou não rejeitada. Caso tenha sido rejeitada a hipótese nula e a amostra seja da distribuição normal multivariada, foi cometido um erro do tipo I. Da mesma forma se a hipótese nula for rejeitada e a amostra foi obtida de uma população não-normal, uma decisão correta foi tomada. Este processo, em cada caso, foi repetido 2.000 vezes e a proporção de decisões incorretas no primeiro caso é a taxa de erro tipo I empírica e, no segundo caso, a proporção de decisões corretas é o poder empírico. Os valores da taxa de erro tipo I empírica foram comparados com o valor nominal por meio de um intervalo de confiança para proporções. Também foram comparadas as taxas de erro e o poder dos dois testes aplicados. Na sequência são descritas todas as etapas necessárias para a simulação das amostras normais e não-normais, aplicação dos testes e cômputo das taxas de erro tipo I e poder. Todas as simulações foram realizadas no programa $\mathbf{R}$.

\section{Taxas de Erro Tipo I}

Foram simuladas amostras aleatórias normais multivariadas de tamanho $\mathrm{n}$ no espaço $\mathrm{p}$-dimensional dadas por $\mathrm{X}_{1}, \mathrm{X}_{2}, \ldots, \mathrm{X}_{\mathrm{n}}$. $\mathrm{O}$ vetor aleatório $\mathrm{X}_{\mathrm{j}}=\left[\mathrm{X}_{\mathrm{j} 1}, \mathrm{X}_{\mathrm{j} 2}, \ldots, \mathrm{X}_{\mathrm{jp}}\right]^{\prime}$ de dimensões $p \times 1$ tem densidade normal multivariada dada por:

$$
f(x)=(2 \pi)^{-p / 2}|\Sigma|^{-1 / 2} \exp \left\{-\frac{1}{2}(x-\mu)^{\prime} \Sigma^{-1}(x-\mu)\right\}
$$

em que $\Sigma$ é matriz de covariância e $\mu$ é o vetor de médias $p \times 1$ populacionais.

Para simular um vetor aleatório $\mathrm{X}_{\mathrm{j}}$ da distribuição apresentada em (1) foi inicialmente obtido o fator de Cholesky $(\Gamma)$ da matriz $\Sigma$, tal que $\Sigma=\Gamma \Gamma^{\prime}$. Posteriormente foi simulado um vetor $Z_{j}$, composto de elementos independentes de uma distribuição normal univariada, sendo que $Z_{j}$ tem distribuição $N_{p}(0, I)$. Utilizando uma transformação linear do vetor dada por $\mathrm{X}_{\mathrm{j}}=\Gamma \mathrm{Z}_{\mathrm{j}}+\mu$, obteve-se o vetor desejado com distribuição dada por (1). Repetiu - se este processo inúmeras vezes para formar a amostra aleatória.

O teste de normalidade multivariado de ShapiroWilk foi aplicado utilizando-se a função mshapiro.test do pacote mvnormtest do programa $\boldsymbol{R}$. Inicialmente foi aplicada uma transformação linear do vetor linear $X_{j}$ dada por $\mathrm{Y}_{\mathrm{j}}=\mathrm{CX}_{\mathrm{j}}$, em que o vetor $\mathrm{C}$ foi determinado a partir de $\mathrm{C}=\mathrm{W}^{-1} \mathrm{R}_{\mathrm{k}}$, sendo $\mathrm{W}^{-1}$ a inversa da matriz de somas de quadrados e produtos amostral e $R_{k} o$ vetor da unidade amostral k contendo os desvios de cada observação para a sua respectiva média. Sendo $\mathrm{R}$ a matriz de desvios de cada observação para a sua média de dimensão $\mathrm{n} \times \mathrm{p}$, a unidade amostral $\mathrm{k}$, selecionada foi considerada a linha da 
matriz $\mathrm{R}$ correspondente ao valor máximo da diagonal da matriz $R W^{-1} R^{\prime}$. As realizações da variável Y são ordenadas de forma crescente obtendo-se $Y_{(1)}, Y_{(2)}, \cdots, Y_{(n)}$ e o teste de Shapiro-Wilk foi aplicado a estes valores. Inicialmente foi obtida a estatística de Shapiro-Wilk por

$$
W=\frac{\left(\sum_{j=1}^{n} \tilde{a}_{j} Y_{(j)}\right)^{2}}{\sum_{j=1}^{n}\left(Y_{j}-\bar{Y}\right)^{2}}
$$

em que os coeficiente $\tilde{a}_{j}$ são os estimadores apresentados por Royston (1983) para os coeficientes populacionais $a_{j}$ relacionadas a j-ésima estatística de ordem da distribuição normal padrão. $\mathrm{O}$ vetor $\mathrm{n} \times 1$ destes coeficientes populacionais são definidos por $a=V^{-1} m /\left(m^{\prime} V^{-2} m\right)$, sendo $m$ o vetor $\mathrm{n} \times 1$ de médias das estatísticas de ordem da normal padrão e $\mathrm{V}$ a matriz $n \times n$ de variâncias e covariâncias das $n$ estatísticas de ordem da normal padrão. Os valores-p são obtidos a partir de uma transformação Box-Cox da estatística do teste, utilizando-se assim uma aproximação normal (ROYSTON, 1983).

Os testes de normalidade baseados nos desvios de assimetria e curtose foram aplicados de acordo com os procedimentos descritos na sequência. Foram utilizadas as definições dos coeficientes multivariados de Mardia $(1970,1974)$ para assimetria $\beta_{1 \mathrm{p}}=\mathrm{E}\left[(\mathrm{X}-\mu)^{\prime} \Sigma^{-1}(\mathrm{Y}-\mu)\right]^{3} \mathrm{e}$ para curtose $\beta_{2 p}=E\left[(X-\mu)^{\prime} \Sigma^{-1}(X-\mu)\right]^{2}$, para uma distribuição p-variada qualquer, para se definir os valores hipotéticos sob normalidade. Assim, sob a hipótese nula de normalidade multivariada estes coeficientes correspondem a $\beta_{1 \mathrm{p}}=0$ e $\beta_{2 \mathrm{p}}=\mathrm{p}(\mathrm{p}+2)$, respectivamente.

Inicialmente foram estimados os coeficientes de assimetria e curtose utilizando os seguintes estimadores:

$$
\hat{\beta}_{1 p}=\frac{1}{n^{2}} \sum_{i=1}^{n} \sum_{j=1}^{n} g^{3} \quad \text { e } \quad \hat{\beta}_{2 p}=\frac{1}{n} \sum_{i=1}^{n} g^{2}{ }_{i i}
$$

em que $g_{i j}=\left(X_{i}-\bar{X} .\right)^{\prime} S_{n}^{-1}\left(X_{j}-\bar{X}.\right), \bar{X} .=\frac{1}{n} \sum_{j=1}^{n} X_{j}$ é o vetor de médias amostrais e $S_{n}=\frac{1}{n} \sum_{j=1}^{n}\left(X_{j}-\bar{X}\right.$. $)\left(X_{j}-\bar{X}\right.$.)' é o estimador viesado de máxima verossimilhança da matriz de covariâncias amostrais.
Em seguida foi aplicado o teste de simetria, cuja estatística, dada por,

$$
\mathrm{k}_{1}=\frac{\mathrm{n} \hat{\beta}_{1 \mathrm{p}}}{6}
$$

possui distribuição assintótica de qui-quadrado com $\mathrm{p}(\mathrm{p}+1)(\mathrm{p}+2) / 6$, graus de liberdade sob a hipótese nula $\mathrm{H}_{0}: \beta_{1 \mathrm{p}}=0$.

Da mesma forma foi aplicado o teste para distribuição mesocúrtica. A estatística do teste dada por

$$
\mathrm{k}_{2}=\frac{\hat{\beta}_{2 \mathrm{p}}-\mathrm{p}(\mathrm{p}+2)}{\sqrt{8 \mathrm{p}(\mathrm{p}+2) / \mathrm{n}}}
$$

possui distribuição assintótica normal padrão sob a hipótese nula .

Se na aplicação dos testes, a hipótese nula for rejeitada a um nível nominal $\alpha$ então, a distribuição dos dados é considerada não-normal.

A proporção de rejeições da hipótese nula foi computada para o teste multivariado de Shapiro-Wilk e para o teste baseado em desvios de simetria e de curtose. As taxas de erro obtidas foram comparadas entre si e com o valor nominal $\alpha$ adotado.

\section{Poder dos testes}

Foram simuladas amostras aleatórias de distribuições não-normais para avaliar o poder dos testes em rejeitar a hipótese nula que por construção é falsa. $\mathrm{O}$ mesmo procedimento para simulação descrito na seção anterior foi aplicado. A diferença consistiu na determinação de diferentes distribuições para os elementos $Z_{\mathrm{i}}$ do vetor $Z$. Foram consideradas amostras aleatórias das distribuições $t$ multivariada com $v=1,2$ e 30 graus de liberdade. Foram utilizadas as funções geradoras de variáveis aleatórias do programa $\mathbf{R}$ para realizar tal tarefa.

Serão aplicados os testes de normalidade descritos anteriormente em 2.000 repetições deste procedimento. A proporção de rejeições da hipótese nula falsa foi computada para o teste multivariado de Shapiro-Wilk e para o teste baseado em desvios de simetria e de curtose. Estas proporções mediram os poderes dos testes, que foram comparados entre si.

\section{RESULTADOS E DISCUSSÃO}

Na Tabela 1 são apresentadas as taxas de erro tipo I dos testes de normalidade baseados em desvios de 
assimetria ( $t a)$ e curtose $(t c)$ e do teste de Shapiro-Wilk $(t s)$ em função da correlação $(\rho)$, tamanho da $\operatorname{amostra}(n)$ e número de variáveis $(p)$. Pode-se observar que, praticamente, não há efeito do $\rho$ nas taxas de erro tipo I, embora exceções existam, como, por exemplo, na situação de $n=100$ e $p=2$, considerando o teste $t c$, que apresentou significância com $\rho=0,5$ e $\rho=0,9$, mas não apresentou diferenças significativas em relação ao nível nominal $\alpha$ $\operatorname{com} \rho=0$.

O tamanho da amostra afeta expressivamente as taxas de erro tipo I de todos os testes. No caso dos testes baseados nos desvios de assimetria e curtose é esperado que quanto maior for a amostra melhor serão os testes, uma vez que suas estatísticas são distribuídas assintoticamente como qui-quadrado e normal,respectivamente. $\mathrm{O}$ teste $t a$ com amostras pequenas tendeu a ser conservativo e passou, em amostras grandes, a apresentar tamanho do teste igual ao valor nominal de $5 \%$. O efeito do aumento do número de variáveis no teste $t a$ foi o de reduzir as taxas de erro tipo I para um mesmo $n$. Assim, para os casos estudados o teste de assimetria apresentou taxas significativamente inferiores ou iguais ao valor nominal. Quanto maior for o número de variáveis recomenda-se aumentar o tamanho da amostra.

O teste de curtose $(t c)$ com amostras pequenas tendeu a ser conservativo, considerando-se um menor número de variáveis envolvidas e tendeu a ser liberal com um maior número de variáveis. Para grandes amostras o mesmo efeito foi observado, exceto que para valores intermediários de $p$ observaram-se taxas de erro similares ao valor nominal $\alpha=5 \%$. Este teste apresenta o inconveniente de ser liberal quando o número de variáveis é grande em relação ao tamanho da amostra utilizado. Assim, por exemplo, para $n=50, p=20$ e $\rho=0,5$ a taxa de erro tipo I é igual a $60,40 \%$.

$\mathrm{O}$ teste de normalidade de Shapiro-Wilk multivariado apresentou o pior desempenho de todos. As taxas de erro tipo I foram sempre superiores ao valor nominal, independentemente de $p$ ou de $n$, chegando a atingir $100 \%$. $\mathrm{O}$ aumento de $n$ provocou uma redução do erro tipo I quando $p$ estava fixado. De forma similar, o aumento de número de variáveis, fixado o valor de $n$, provocou um grande aumento na taxa do erro tipo I. A taxa de crescimento do erro tipo I em função de $p$ foi muito grande, fazendo com que houvesse casos com taxa de $100 \%$. Assim, o teste de normalidade de Shapiro-Wilk multivariado implementado no programa $\mathbf{R}$ é extremamente liberal e a sua aplicação é desaconselhada.

Para as taxas de erro tipo I dos três testes, considerando $\alpha=1 \%$ (resultados não apresentados), praticamente as mesmas constatações foram feitas. O teste de assimetria foi conservativo ou de mesmo tamanho do nível nominal. O teste de curtose foi conservativo para pequenas amostras e pequenos valores de $p$ e liberal com grandes valores de $n$ e $p$. O teste de normalidade de Shapiro-Wilk multivariado foi sempre liberal e com pior desempenho quando $p$ era grande com relação a $n$. Não houve efeito da estrutura de correlação, da mesma forma como foi observado para $\alpha=5 \%$ (Tabela 1). Para $\alpha=1 \%$ o teste de normalidade de Shapiro-Wilk multivariado implementado no programa $\mathbf{R}$ também não deve ser recomendado.

\section{Poder}

Na Tabela 2 estão apresentados os valores de poder dos testes $t a, t c$ e $t s$ em função de diferentes valores de $n$, $p$ e $\rho$ para $\alpha=5 \%$. Foi considerada a distribuição $t$ de Student com $v=1$ grau de liberdade, criando uma situação de grande afastamento da normalidade para $\alpha=5 \%$ (Tabela 2). Conforme aconteceu com as taxas de erro tipo I praticamente não houve efeito da correlação $r$ nos valores de poder. Para o teste de Shapiro-Wilk os valores de poder foram quase sempre elevados $(>80 \%)$ e na maioria das vezes iguais a $100 \%$. Infelizmente, estes valores não são reais, pois o teste não controlou as taxas de erro tipo I, sendo sempre liberal. Para este teste o poder cresceu rapidamente com o aumento do número de variáveis atingindo $100 \%$, mesmo em situações de pequenas amostras como, por exemplo, para $n=10$ e $p=6$. Os testes de assimetria e curtose nesta mesma situação apresentaram valores de poder nulos, independentemente de $\rho$. Assim, não se aconselha a comparação do poder do teste $t s$ com os demais e não se recomenda seu uso para testar a normalidade multivariada.

O teste de desvios de curtose $(t c)$ apresentou valores de poder bastante elevados para $n$ superior a 20 , mas foi severamente afetado pelo número de variáveis. Quanto maior for o valor de $p$ para $n \leq 20$, menor é o poder deste teste. Este resultado é surpreendente, pois o erro tipo I nestas condições foi significativamente $(p<0,01)$ superior aos valores nominais (Tabela 1) de 5\% e também de1\% (resultados não apresentados). Ocorreu exatamente o contrário, o poder foi relativamente mais baixo que o esperado. Para $n \geq 50$ este efeito não foi observado, embora o erro tipo I continuasse significativamente superior a $\alpha$.

O teste baseado em desvios de simetria $(t a)$ apresentou valores de poder elevados (>90\%) para $n \geq 20 \mathrm{e}$ para quaisquer valores de $p$. O teste $t a$ para $n \geq 50$ foi bastante conservativo e com isso eram esperados valores 
Tabela 1 -Taxas de erro tipo I dos três testes de normalidade multivariados: teste de assimetria ( $t a)$, teste de curtose (tc) e teste de Shapiro-Wilk (ts) e $\alpha=5 \%$.

\begin{tabular}{|c|c|c|c|c|c|c|c|c|c|c|}
\hline \multirow[b]{2}{*}{$n$} & \multirow[b]{2}{*}{$p$} & \multicolumn{3}{|c|}{$\rho=0,0$} & \multicolumn{3}{|c|}{$\rho=0,5$} & \multicolumn{3}{|c|}{$\rho=0,9$} \\
\hline & & $t a$ & $t c$ & ts & $t a$ & $t c$ & ts & $t a$ & $t c$ & $t s$ \\
\hline \multirow{3}{*}{10} & 2 & 0,25 & 0,00 & 16,80 & 0,55 & 0,00 & 17,85 & 0,50 & 0,00 & 17,40 \\
\hline & 4 & 0,00 & 0,00 & 79,85 & 0,00 & 0,00 & 77,70 & 0,00 & 0,00 & 79,40 \\
\hline & 6 & 0,00 & 0,00 & 100,00 & 0,00 & 0,00 & 100,00 & 0,00 & 0,00 & 100,00 \\
\hline \multirow{4}{*}{20} & 2 & 2,55 & 0,70 & 14,35 & 2,55 & 0,40 & 11,50 & 3,25 & 0,85 & 13,85 \\
\hline & 4 & 1,70 & 0,30 & 58,25 & 1,45 & 0,35 & 59,50 & 1,60 & 0,15 & 56,40 \\
\hline & 6 & 0,60 & 1,75 & 97,05 & 0,35 & 1,75 & 97,70 & 0,45 & 1,60 & 97,30 \\
\hline & 10 & 0,00 & 19,40 & 100,00 & 0,00 & 20,45 & 100,00 & 0,00 & 20,15 & 100,00 \\
\hline \multirow{5}{*}{50} & 2 & 3,35 & 1,90 & 10,50 & 4,20 & 1,80 & 11,20 & 4,30 & 1,75 & 11,25 \\
\hline & 4 & 3,25 & 1,80 & 43,60 & 3,95 & 1,75 & 43,70 & 3,95 & 2,20 & 47,40 \\
\hline & 6 & 3,15 & 2,85 & 89,20 & 3,55 & 2,95 & 87,80 & 3,30 & 2,90 & 88,40 \\
\hline & 10 & 2,15 & 8,05 & 100,00 & 1,35 & 9,60 & 100,00 & 2,35 & 9,00 & 100,00 \\
\hline & 20 & 0,30 & 59,55 & 100,00 & 0,10 & 60,40 & 100,00 & 0,00 & 60,80 & 100,00 \\
\hline \multirow{5}{*}{100} & 2 & 4,20 & 3,40 & 9,85 & 4,65 & 3,00 & 10,35 & 4,40 & 2,80 & 10,00 \\
\hline & 4 & 5,65 & 2,15 & 40,20 & 5,10 & 3,40 & 38,80 & 4,55 & 2,60 & 38,60 \\
\hline & 6 & 4,80 & 4,40 & 82,55 & 4,50 & 4,40 & 80,50 & 4,35 & 3,50 & 81,80 \\
\hline & 10 & 3,80 & 6,80 & 100,00 & 3,15 & 8,20 & 100,00 & 3,10 & 5,95 & 100,00 \\
\hline & 20 & 1,85 & 26,30 & 100,00 & 1,35 & 25,70 & 100,00 & 1,80 & 26,65 & 100,00 \\
\hline \multirow{5}{*}{200} & 2 & 4,80 & 3,85 & 11,20 & 5,70 & 3,70 & 9,90 & 4,40 & 3,50 & 9,10 \\
\hline & 4 & 5,15 & 4,65 & 37,20 & 5,65 & 4,55 & 38,25 & 5,10 & 3,50 & 35,85 \\
\hline & 6 & 4,75 & 4,45 & 77,10 & 5,10 & 3,85 & 76,65 & 5,20 & 4,35 & 78,40 \\
\hline & 10 & 5,20 & 5,85 & 100,00 & 4,95 & 5,85 & 100,00 & 4,90 & 5,70 & 100,00 \\
\hline & 20 & 3,50 & 14,60 & 100,00 & 3,35 & 14,05 & 100,00 & 2,75 & 13,15 & 100,00 \\
\hline
\end{tabular}

baixos de poder, porém isso não ocorreu. A exceção ocorreu para amostras pequenas como $n=10$ e principalmente maior número de variáveis $(p \geq 4)$ Com $n=10, p=2$ e $\rho=0$ o poder do teste $t a$ foi de $54,45 \%$, o que pode ser considerado relativamente satisfatório. Para $p=6$, com o mesmo tamanho de $n$, o poder foi nulo. Com amostras de tamanho $n=200$ todos os valores de poder foram iguais a $100 \%$.

Os valores de poder para os testes em função de $n$, $p$ e $\rho$ com $\alpha=1 \%$, considerando a distribuição $t$ de Student com $v=1$ teve o mesmo padrão de resposta apontado anteriormente para $\alpha=5 \%$ (Tabela 2). O que ocorre, no entanto, como já era esperado, é que os valores de poder são menores do que aqueles observados para $\alpha=5 \%$. Em todos os casos em que $n \geq 20$, o poder foi superior a $85 \%$, exceto para $t c \operatorname{com} n=20$ e $p=10$ em que os valores eram de aproximadamente de $30 \%$. Houve uma tendência do poder do teste $t a$ ser superior ao do teste $t c$. Com $n=200$ todos os valores foram iguais a $100 \%$.

Na Tabela 3 estão apresentados os valores de poder dos três testes estudados para $\alpha=1 \%$ e $5 \%$ em função de $p, n$ e $\rho$. Apenas valores de $n=20$ e 200 foram considerados, uma vez que os padrões anteriores se repetiram nesta situação na qual foi considerada a distribuição $t \operatorname{com} v=30$ graus de liberdade. Os valores de poder dos testes $t a$ e $t c$ foram muito pequenos $(\leq \alpha)$ para $n=20$. Houve algumas exceções para o teste $t c$ dados pela situação de $p=10$. Novamente pode-se observar que o teste $t s$ foi o mais poderoso e que o poder aumentou com aumento de $p$. Neste caso particular de $n=20$, o poder de $100 \%$ foi atingido com $p=10$ mesmo para $\alpha=1 \%$. Novamente deve-se afirmar que os grandes valores de poder encontrados para o teste $t s$ não devem ser considerados, pois o seu tamanho é significativamente e expressivamente superior ao valor nominal $\alpha$.

Para $n=200$ os valores de poder foram classificados na seguinte ordem $t a<t c<t s$ para todas as combinações de $p, \rho$ e $\alpha$. A comparação dos valores de poder correspondentes às distribuições $t$ com $v=1$ e 30 graus de liberdade (Tabelas 2 e 3 ) permite que se observe uma drástica redução com o aumento dos graus de liberdade 
Tabela 2 - Poder dos três testes de normalidade multivariados: teste de assimetria $(t a)$, teste de curtose $(t c)$ e teste de Shapiro-Wilk (ts) para a distribuição $t$ com $v=1$ grau de liberdade e $\alpha=5 \%$.

\begin{tabular}{|c|c|c|c|c|c|c|c|c|c|c|}
\hline \multirow[b]{2}{*}{$n$} & \multirow[b]{2}{*}{$p$} & \multicolumn{3}{|c|}{$\rho=0,0$} & \multicolumn{3}{|c|}{$\rho=0,5$} & \multicolumn{3}{|c|}{$\rho=0,9$} \\
\hline & & $t a$ & $t c$ & $t s$ & $t a$ & $t c$ & $t s$ & $t a$ & $t c$ & $t s$ \\
\hline \multirow{3}{*}{10} & 2 & 54,45 & 16,85 & 82,00 & 54,90 & 16,20 & 82,40 & 54,30 & 16,00 & 82,45 \\
\hline & 4 & 20,50 & 0,00 & 99,00 & 21,05 & 0,00 & 99,20 & 21,60 & 0,00 & 99,50 \\
\hline & 6 & 0,00 & 0,00 & 100,00 & 0,00 & 0,00 & 100,00 & 0,00 & 0,00 & 100,00 \\
\hline \multirow{4}{*}{20} & 2 & 92,40 & 91,70 & 97,50 & 91,90 & 91,05 & 97,20 & 93,00 & 93,00 & 97,00 \\
\hline & 4 & 98,40 & 96,20 & 99,90 & 98,55 & 96,80 & 100,00 & 98,55 & 96,75 & 99,95 \\
\hline & 6 & 99,40 & 96,15 & 100,00 & 99,35 & 95,80 & 100,00 & 99,25 & 96,05 & 100,00 \\
\hline & 10 & 97,70 & 51,45 & 100,00 & 97,55 & 52,30 & 100,00 & 97,20 & 51,20 & 100,00 \\
\hline \multirow{5}{*}{50} & 2 & 99,75 & 100,00 & 99,95 & 99,50 & 100,00 & 99,95 & 99,50 & 100,00 & 100,00 \\
\hline & 4 & 100,00 & 100,00 & 100,00 & 100,00 & 100,00 & 100,00 & 100,00 & 100,00 & 100,00 \\
\hline & 6 & 100,00 & 100,00 & 100,00 & 100,00 & 100,00 & 100,00 & 100,00 & 100,00 & 100,00 \\
\hline & 10 & 100,00 & 100,00 & 100,00 & 100,00 & 100,00 & 100,00 & 100,00 & 100,00 & 100,00 \\
\hline & 20 & 100,00 & 100,00 & 100,00 & 100,00 & 100,00 & 100,00 & 100,00 & 100,00 & 100,00 \\
\hline \multirow{5}{*}{200} & 2 & 100,00 & 100,00 & 100,00 & 100,00 & 100,00 & 100,00 & 100,00 & 100,00 & 100,00 \\
\hline & 4 & 100,00 & 100,00 & 100,00 & 100,00 & 100,00 & 100,00 & 100,00 & 100,00 & 100,00 \\
\hline & 6 & 100,00 & 100,00 & 100,00 & 100,00 & 100,00 & 100,00 & 100,00 & 100,00 & 100,00 \\
\hline & 10 & 100,00 & 100,00 & 100,00 & 100,00 & 100,00 & 100,00 & 100,00 & 100,00 & 100,00 \\
\hline & 20 & 100,00 & 100,00 & 100,00 & 100,00 & 100,00 & 100,00 & 100,00 & 100,00 & 100,00 \\
\hline
\end{tabular}

de 1 para 30. Isso ocorreu por causa da aproximação da distribuição $t$ multivariada à normal multivariada quando $v \rightarrow \infty$. A superioridade do teste $t c$ em relação ao teste $t a$ provavelmente possa ser explicada pela aproximação mais lenta da mesocurtose na medida que o parâmetro $v$ aumenta.

\section{Considerações finais}

O programa R (R DEVELOPMENT CORE TEAM, 2006) tem alcançado grande popularidade entre os pesquisadores das mais diferentes áreas da ciência. Este programa possui código fonte aberto e recebe contribuições de profissionais do mundo todo. Estas contribuições são denominadas de pacotes e muitas vezes são avaliadas e recomendadas pelo grupo de desenvolvimento do programa.

O teste de Shapiro-Wilk generalizado para o caso multivariado é um desses pacotes. Este teste teve uma pobre performance nos estudos de simulação realizados neste trabalho. $\mathrm{O}$ erro tipo I deste teste foi elevado e muito superior aos valores nominais adotados. Esse resultado foi avaliado em várias situações e as taxas de erro tipo I deste teste cresceram na medida que o número de variáveis cresceu, atingindo $100 \%$ com valores moderados de $p$. O poder, por outro lado, foi quase sempre superior ao dos testes concorrentes. Isso, no entanto, não pode ser considerado, pois o tamanho do teste foi superior ao nível nominal $\alpha$.

Os testes de desvios de assimetria e de curtose mostraram-se como alternativas eficientes para avaliar a hipótese nula de normalidade. Por se tratarem de testes assintóticos, foi recomendado por Santos \& Ferreira (2003) utilizar $n \geq 50$ para assimetria e $n \geq 100$ para curtose.

Em função desses resultados, recomendam-se avaliações científicas rotineiras de procedimentos de estimação e de testes de hipóteses implementados como pacotes do programa R. Para o caso particular do teste de Shapiro-Wilk multivariado não se recomenda a utilização da função mshapiro.test do pacote mvnormtest deste programa. 
Tabela 3 - Poder dos três testes de normalidade multivariados: teste de assimetria $(t a)$, teste de curtose $(t c)$ e teste de Shapiro-Wilk (ts) para a distribuição $t$ com $v=30$ graus de liberdade e $\alpha=5 \%$ e $1 \%$.

\begin{tabular}{|c|c|c|c|c|c|c|c|c|c|c|}
\hline \multirow[b]{3}{*}{$n$} & \multirow[b]{3}{*}{$p$} & \multicolumn{8}{|c|}{$\alpha=5 \%$} & \\
\hline & & \multicolumn{3}{|c|}{$\rho=0,0$} & \multicolumn{3}{|c|}{$\rho=0,5$} & \multicolumn{3}{|c|}{$\rho=0,9$} \\
\hline & & $t a$ & $t c$ & $t s$ & $t a$ & $t c$ & $t s$ & $t a$ & $t c$ & $t s$ \\
\hline \multirow{4}{*}{20} & 2 & 4,45 & 0,90 & 16,15 & 5,15 & 1,65 & 17,55 & 5,10 & 1,20 & 17,50 \\
\hline & 4 & 3,40 & 0,20 & 65,70 & 3,80 & 0,00 & 65,30 & 2,90 & 0,25 & 64,70 \\
\hline & 6 & 1,40 & 1,00 & 98,45 & 2,50 & 0,40 & 98,35 & 1,35 & 0,80 & 98,50 \\
\hline & 10 & 0,00 & 13,20 & 100,00 & 0,00 & 11,95 & 100,00 & 0,00 & 1,21 & 100,00 \\
\hline \multirow{5}{*}{200} & 2 & 12,70 & 19,10 & 24,35 & 11,45 & 17,30 & 22,75 & 12,20 & 17,00 & 23,10 \\
\hline & 4 & 21,40 & 30,80 & 67,40 & 22,30 & 32,15 & 67,75 & 20,20 & 31,35 & 65,20 \\
\hline & 6 & 31,60 & 46,80 & 96,55 & 30,25 & 44,35 & 95,60 & 31,75 & 43,60 & 95,70 \\
\hline & 10 & 58,20 & 72,00 & 100,00 & 56,20 & 72,70 & 100,00 & 57,00 & 73,80 & 100,00 \\
\hline & 20 & 95,70 & 96,75 & 100,00 & 89,50 & 90,60 & 93,35 & 95,05 & 97,00 & 100,00 \\
\hline \multicolumn{11}{|c|}{$\alpha=1 \%$} \\
\hline \multirow[b]{2}{*}{$n$} & & \multicolumn{3}{|c|}{$\rho=0,0$} & \multicolumn{3}{|c|}{$\rho=0,5$} & \multicolumn{3}{|c|}{$\rho=0,9$} \\
\hline & $p$ & $t a$ & $t c$ & $t s$ & $t a$ & $t c$ & $t s$ & $t a$ & $t c$ & $t s$ \\
\hline \multirow{4}{*}{20} & 2 & 1,10 & 0,25 & 4,70 & 1,50 & 0,35 & 6,45 & 1,30 & 0,40 & 6,45 \\
\hline & 4 & 0,70 & 0,00 & 35,85 & 0,90 & 0,00 & 35,95 & 0,60 & 0,00 & 34,75 \\
\hline & 6 & 0,20 & 0,00 & 86,20 & 0,70 & 0,00 & 84,40 & 0,15 & 0,00 & 83,90 \\
\hline & 10 & 0,00 & 0,00 & 100,00 & 0,00 & 0,00 & 100,00 & 0,00 & 0,00 & 100,00 \\
\hline \multirow{5}{*}{200} & 2 & 5,55 & 10,85 & 11,35 & 4,10 & 9,05 & 8,95 & 4,25 & 8,65 & 10,15 \\
\hline & 4 & 9,75 & 19,15 & 41,10 & 9,50 & 20,20 & 43,65 & 8,95 & 17,45 & 39,60 \\
\hline & 6 & 16,40 & 30,75 & 80,95 & 16,15 & 28,65 & 79,65 & 15,55 & 28,15 & 79,50 \\
\hline & 10 & 39,10 & 55,95 & 100,00 & 36,75 & 56,05 & 100,00 & 38,90 & 56,40 & 100,00 \\
\hline & 20 & 89,85 & 91,95 & 100,00 & 83,00 & 85,55 & 93,35 & 89,05 & 91,30 & 100,00 \\
\hline
\end{tabular}

\section{CONCLUSÕES}

O teste de Shapiro-Wilk multivariado do programa $\mathbf{R}$, função mshapiro.test do pacote mvnormtest, tem fraco desempenho e não é recomendado para uso rotineiro.

O teste de normalidade baseado em desvios de assimetria e curtose é recomendado para situações de $n \geq 50$ e $n \geq 100$, respectivamente.

\section{REFERÊNCIAS BIBLIOGRÁFICAS}

DOMANSKI, C. Wlasnosci testu wielowymiarowej normalnosci Shapiro-Wilka i jego zastosowanie. Cracow: University of Economics Rector's Lectures, 1998.

MARDIA, K. V. Measures of multivariate skewness and kurtosis with applications. Biometrika, London, v. 57, n. 3, p. 519-530, 1970.

MARDIA, K. V. Applications of some measures of multivariate skewness and kurtosis for testing normality and robustness studies. Sankhyã A, [S.1.], v. 36, p. 115-128, 1974.

MARDIA, K. V. Assessment of multinormality and the robustness of Hotelling's $\mathrm{T}^{2}$ test. Applied Statistics, London, v. 24, n. 2, p. 163-171, 1975.

R DEVELOPMENT CORE TEAM. A language and environment for statistical computing. Vienna: $R$ Foundation for Statistical Computing, 2006.

ROYSTON, J. B. Some techniques for assessing multivariate based on the Shapiro-Wilk W. Applied Statistics, London, v. 32, n. 2, p. 121-133, 1983.

SANTOS, A. C.; FERREIRA, D. F. Definição do tamanho amostral usando simulação Monte Carlo para o teste de normalidade baseado em assimetria e curtose: II. Abordagem multivariada. Ciência e Agrotecnlogia, Lavras, v. 24, n. 1, p. 62-69, 2003. 